\title{
Connectorisation of fibre Bragg grating sensors recorded in microstructured polymer optical fibre
}

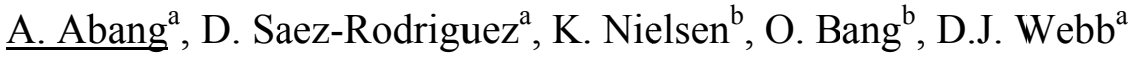 \\ ${ }^{a}$ Aston Institute of Photonic Technologies, Aston University, Birmingham, B4 7ET, UK \\ ${ }^{b}$ Department of Photonics Engineering, Technical University of Denmark, Ørsteds Plads, 2800, Kgs. \\ Lyngby, Denmark
}

\begin{abstract}
We describe the production and characterization of FC/PC connectorised fibre Bragg grating sensors in polymer fibre. Sensors were recorded in few-moded and single mode microstructured fibre composed of poly (methyl methacrylate).
\end{abstract}

Keywords: POF, FBG, sensor

\section{INTRODUCTION}

Fibre Bragg grating sensors recorded in polymer optical fibre (POF) have existed for more than 10 years, however only recently has their use started to be explored by industry. One of the barriers to their exploitation has been the difficulty of connecting the POF to a silica fibre connecting lead. One solution has been to glue the two fibres together ${ }^{1}$, though this does not permit demountable connections being formed. In this paper we describe procedures to enable connectorisation of microstructured POF (mPOF) containing fibre Bragg grating (FBG) sensors. Following on from our recent work with multimode $\mathrm{mPOF}$ sensors ${ }^{2}$, for the first time demountable connections with single mode POF incorporating gratings have been made.

\section{MULTIMODE MPOF CONNECTORISATION}

\subsection{Inscription}

Initial tests were carried out on a poly(methyl methacrylate) (PMMA) based multimode mPOF fibre (Kiriama Pty Ltd.). This had an outer diameter of $150 \mu \mathrm{m}$ and a core diameter of $50 \mu \mathrm{m}$, the core of the fibre being bounded by 3 rings of holes having a diameter of $5 \mu \mathrm{m}$ and spaced $5 \mu \mathrm{m}$ from each other - see figure1. The low average refractive index of the ring of holes effectively provides index guiding in the solid core of the fibre.

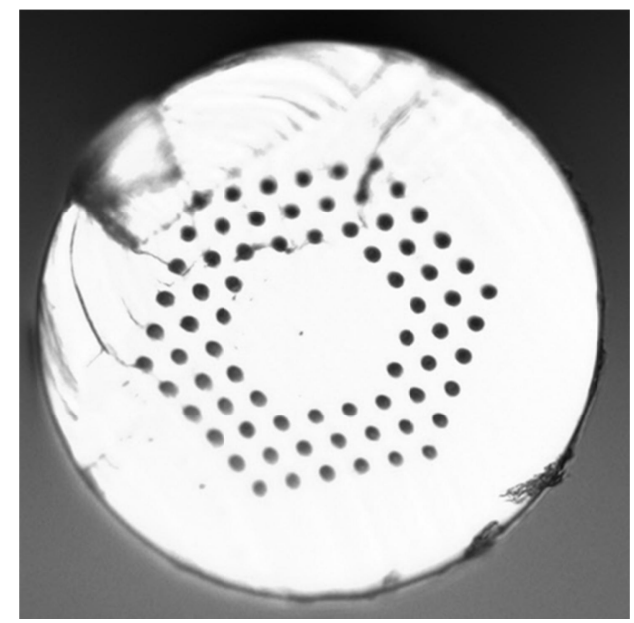

Fig. 1 Microscope image of end face of multimode $\mathrm{mPOF}$

Fifth European Workshop on Optical Fibre Sensors, edited by Leszek R. Jaroszewicz, Proc. of SPIE Vol. 8794, 87943Q · (c) 2013 SPIE · CCC code: 0277-786X/13/\$18 · doi: 10.1117/12.2026796 
A helium-cadmium laser with a wavelength of $325 \mathrm{~nm}$ and an ultraviolet (UV) power output of $30 \mathrm{~mW}$ was used to inscribe the Bragg grating in the mPOF. The laser beam was focused vertically downwards using a $10 \mathrm{~cm}$ focal length cylindrical lens, through a $557.5 \mathrm{~nm}$ period phase mask and on to the fibre. The multimode mPOF was laid on a v-groove and taped down using polyimide tape. The inscription time of this fibre requires between 40-60 minutes. The Bragg wavelength of the inscribed grating was in the region of $830 \mathrm{~nm}$ with a grating length of $2 \mathrm{~mm}$, determined by the width of the UV laser beam, and a full width at half maximum bandwidth of $0.3 \mathrm{~nm}$.

Inscription was monitored using an 830nm multimode mode 50:50 silica fibre coupler (Thorlabs), a 2mW SLD light source (Superlum SLD371, bandwidth 50nm) and an optical spectrum analyzer (OSA Hewlett Packard 70004A). A connection was made between the arm of the silica coupler and the mPOF using an FC/APC connector on the silica fibre, which was butt coupled to the POF. The end faces of the POF were cleaved using an $80^{\circ} \mathrm{C}$ hot cleave as described by Abdi et $\mathrm{al}^{3}$. A small amount of polymer index matching gel was used in the coupling to reduce Fresnel reflections.

\subsection{Etching of the fibre}

Acetone etching ${ }^{4}$ was applied to one end of the $150 \mu \mathrm{m}$ diameter multimode mPOF for 4 minutes to reduce its size and enable it to fit into the $128 \mu \mathrm{m}$ ceramic ferrule connector. The diameter of the end of the mPOF after etching was $121 \mu \mathrm{m}$. The etched part of the fibre was inserted into the ferrule of an FC/PC connector and pulled out gently from the other side until it jammed in the ferrule connector. Norland 76 UV curing adhesive was applied on the fibre and the fibre was pulled back and forth so that the glue was drawn into the ferrule. The protruding end of the fibre was cleaved using a hot $\left(80^{\circ} \mathrm{C}\right)$ blade. The cleaved end was UV glued with the UV curing lamp for $10 \mathrm{~min}$. The curing lamp had a $2 \%$ iris coverage which provided an optical output power of $60 \mathrm{~mW}$. The lamp was positioned $3 \mathrm{~cm}$ away from the polished face of the ferrule connector at an approximate angle of 45 degrees for effective curing.

\section{2 mPOF Connector Polishing}

Four different aluminium oxide polishing papers of $5,3,1$, and $0.3 \mu \mathrm{m}$ were used to polish the UV glued end of the $\mathrm{mPOF}$ connector. Two polishing cycles were carried out using the polishing papers in descending order of grit size. The polishing papers were placed one after the other on a rubber polishing pad (Thorlabs NRS913) which was placed on a glass polishing plate. The glass plate served as a flat and stable surface for easy polishing, while the rubber polishing pad helped to protect the polymer fibre from excessive force due to the hardness of the glass plate. The glued face of the multimode mPOF ferrule connector was inserted into a polishing disc (Thorlabs D50-FC) and polished slowly in a figure-eight pattern ${ }^{5}$ to reduce the heat generated which could otherwise melt the polymer fibre - see figure $2 \mathrm{a}$. The polishing disc helped to keep the tip of the ferrule normal to the paper while polishing. The glass plate and polishing disc were cleaned between polishing steps with lab tissue soaked in isopropyl alcohol. This was done to remove any particles that could damage the face of the fibre. The face of the connector was inspected repeatedly during the two polishing cycles using a hand-held microscope (Priorspec II). Figure 2b shows the polished end-face of the mPOF connector which was captured using a Zeiss Axioskop 2 microscope.
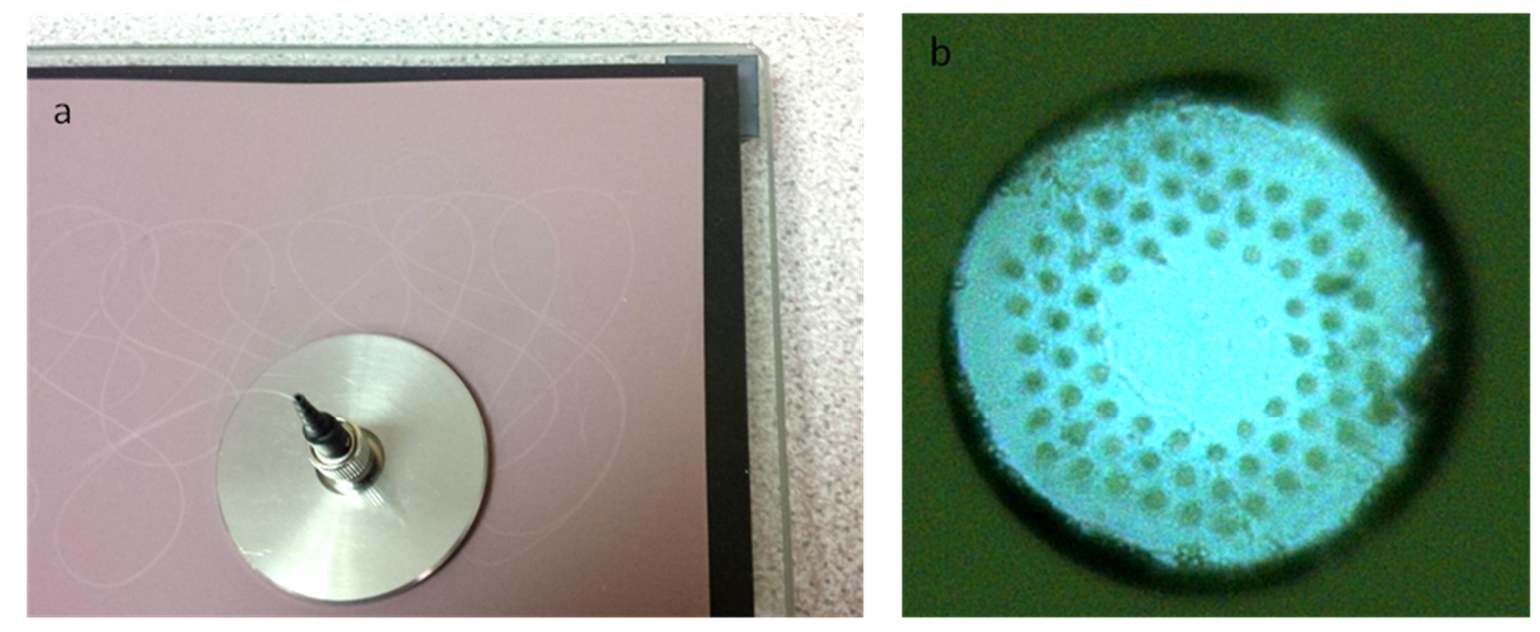

Figure 2(a) Fibre polishing process (b) mPOF in ferrule connector after polishing 
After polishing, the multimode mPOF grating was connected via a conventional bulkhead connector to a 50 micron core, multimode, step index 830nm 50:50 silica coupler. The SLD light source and OSA described above were connected to the other two ends of the silica coupler. The reflection of the grating was captured on the OSA as shown in figure 3 . The total length of fibre in the connector was $12 \mathrm{~cm}$. The FBG was $7.5 \mathrm{~cm}$ away from the polished end and $4.3 \mathrm{~cm}$ from the butt-coupled end. The response obtained by interrogating the grating using the cleaved non-connectorised end of the fibre was approximately the same as that using the connector, despite their varying distance from the FBG, indicating that the coupling loss at the connector was possibly lower than that achieved with butt coupling.

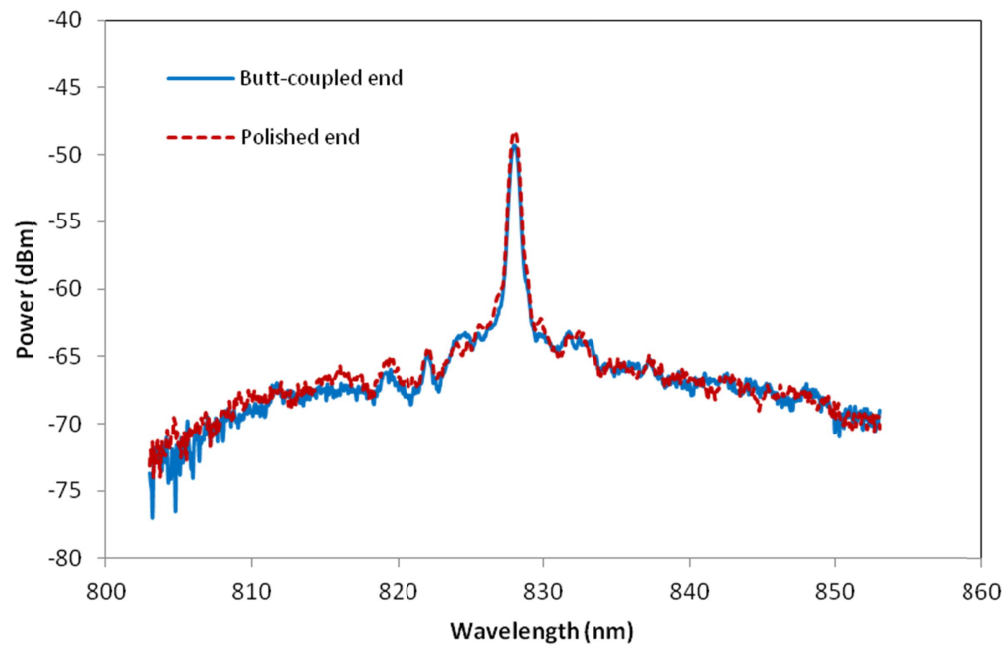

Figure 3 Reflection spectrums of FBG from the polished end and the butt-coupled end of fibre

\section{SINGLEMODE MPOF CONNECTORISATION}

In this section we report on a study of the losses in the connection of a single mode microstructure polymer optical fibre (mPOF) with a single mode silica fibre using a FC/PC connector. The mPOF was fabricated in the Department of Photonics Engineering at the Technical University of Denmark by drilling a solid PMMA preform. The fibre possessed a three ring hole structure. Its diameter was $135 \pm 5 \mu \mathrm{m}$ and the average pitch and hole diameter were $\Lambda=4.26 \mu \mathrm{m}$ and $\mathrm{d}=1.87 \mu \mathrm{m}$, so the ratio is $\mathrm{d} / \Lambda=0.44$; therefore it is expected that the polymer fibre is single mode ${ }^{6}$.

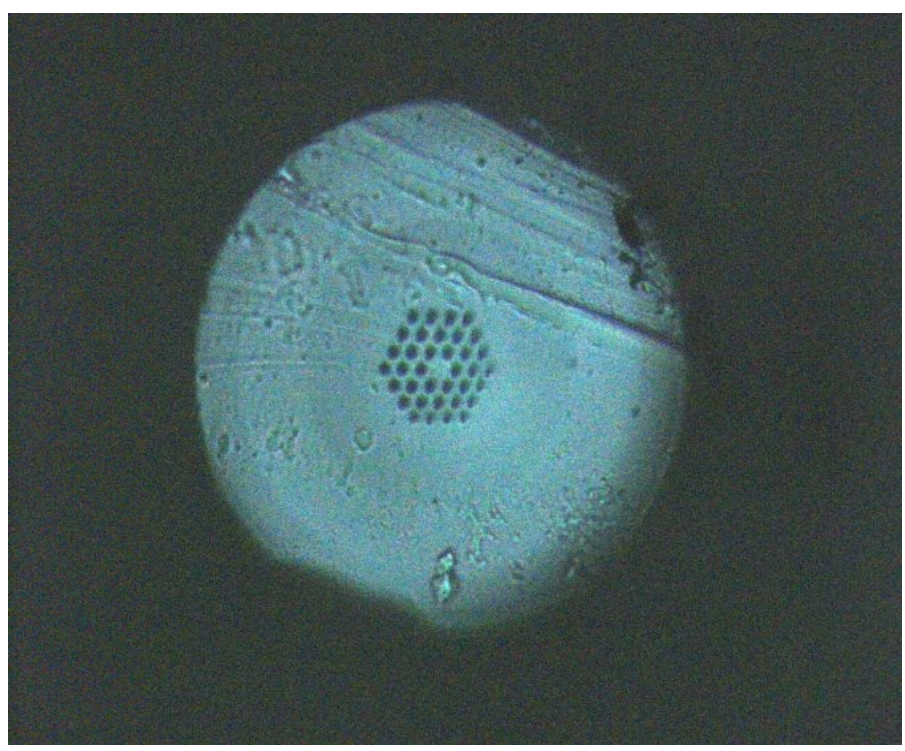

Figure 4. Fibre cut in the connector. The background is the ferrule. 
First, the mPOF was connectorised using a FC connector with a $120 \mu \mathrm{m}$ diameter ferrule. As a consequence, once again the mPOF had to be etched using acetone for several seconds (from 10 to 15 seconds, depending on the exact fibre diameter) to reduce the diameter. Once the fibre diameter was less than the ferrule bore it was introduced in the connector and it was pulled out until the fibre became fitted. Finally the excess fibre in the end of the connector was cleaved $^{7}$ with a hot blade at $77 \mathrm{C}$ along the ferrule surface of the connector. Figure 4 show the section of the fibre in the ferrule bore. This cleaving process has proven to be just as reliable as the polishing method described in the previous section, but is much quicker to implement.

The cut-back method was used to measure the connection losses. A red laser with a maximum power of $620 \mu \mathrm{W}$ at 636 $\mathrm{nm}$ was used as a source. It had its output coupled to a single mode silica fibre with an end FC connector; its output was connected to the connectorised mPOF and the transmitted power was measured for several lengths. The transmitted power of a fibre, including the connector losses, can be written as:

$$
P=P_{0} \cdot e^{-\alpha_{1} \cdot L-\alpha_{2}}
$$

where $\mathrm{P}$ is the transmitted power over a length of fibre $\mathrm{L}$ and $\mathrm{P}_{0}$ the power of the laser. Finally, $\alpha_{1}$ and $\alpha_{2}$ are the loss coefficients of the fibre and the connector respectively. If we rearrange equation (1) we obtain the following linear expression.

$$
\operatorname{Ln}\left(\frac{P}{P_{0}}\right)=-\alpha_{1} \cdot L-\alpha_{2}
$$

In figure 5 are represented both the experimental data and their linear fit.

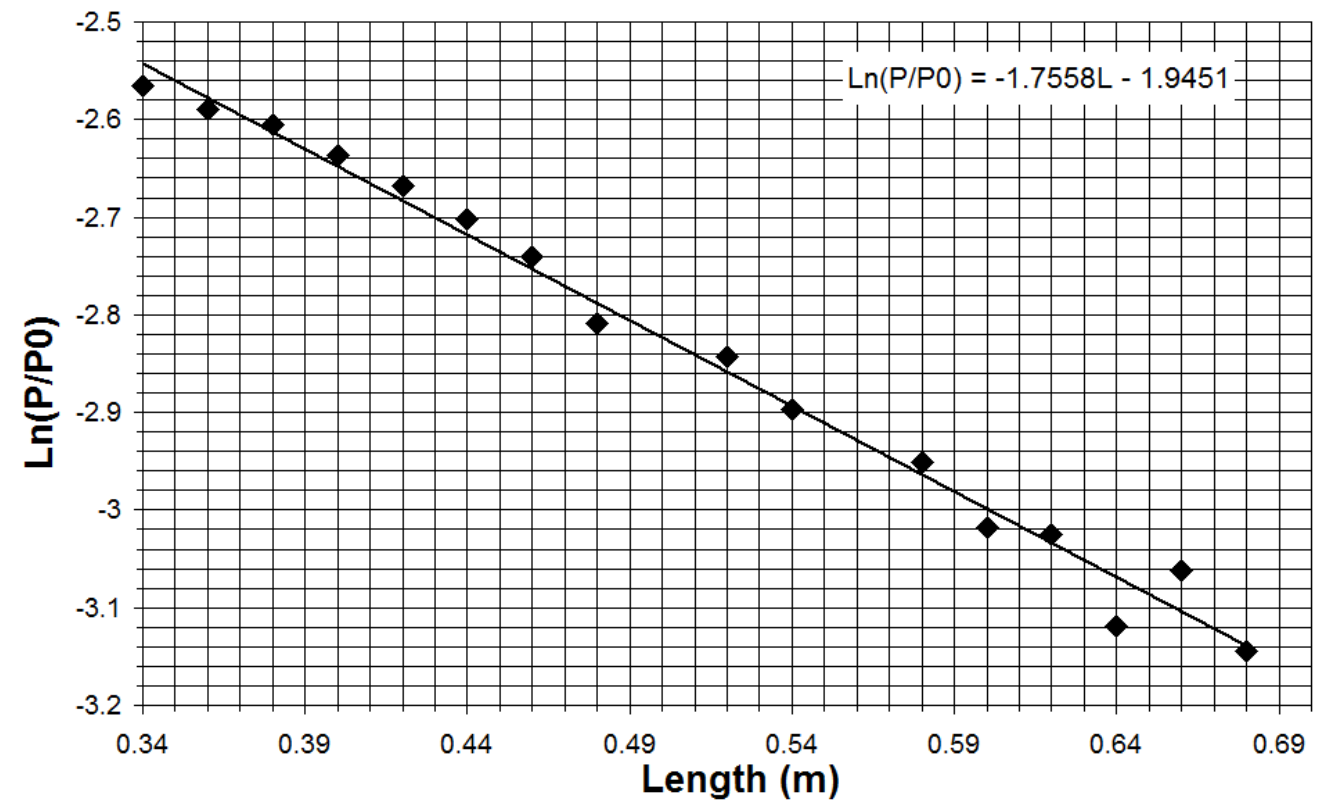

Figure 5. Cut-back measurements of transmitted power. The dots are the experimental data, and the line is the linear fit.

According to equation (2), the slope and the coordinate origin are the loss coefficient of the fibre and the loss of the connection respectively. In table 1 are given the losses with their errors both in linear and logarithmic scales, as measured at $636 \mathrm{~nm}$. 
Table 1. Connector and fiber losses at $636 \mathrm{~nm}$.

\begin{tabular}{|l|l|l|l|}
\hline$\alpha_{1}\left(\mathbf{m}^{-1}\right)$ & $\alpha_{2}$ & $\alpha_{1 \mathbf{d B}}\left(\mathbf{d B m}^{-1}\right)$ & $\alpha_{2 \mathbf{d B}}(\mathbf{d B})$ \\
\hline $1.76 \pm 0.05$ & $1.95 \pm 0.03$ & $7.63 \pm 0.22$ & $8.45 \pm 0.12$ \\
\hline
\end{tabular}

\section{DISCUSSION}

In our initial experiments with connectorised grating sensors, it appears that the signal to noise ratio obtained is at least as good as is the case when a glued connection is used between silica and POF. Consequently, we believe that these demountable connectors offer an enhancement to the usability of the polymer grating sensor technology. We also believe that further optimization of the connectorisation process should result in significant improvements to the losses obtained, which are still well in excess of what modeling suggests, taking into account the different mode field profiles and effective indices in the silica fibre and POF. Of the two approaches to preparing the fibre end in the connector, the cleaving method is simpler and much quicker to implement than the polishing approach, however we have not yet carried out a detailed study of the coupling efficiency achieved with each technique. Another important issue not yet properly studied will be the lifetime of such connectors, in terms of number of connections that can be made before the fibre surface becomes damaged. A small number of reconnections results in no significant changes to the device behavior but clearly further study of the robustness of the devices is needed - particularly in the case of the single mode connectors.

\section{ACKNOWLEDGEMENT}

This work is supported by a Marie Curie Intra European Fellowship included in the 7th Framework Program of the European Union (project PIEF-GA-2011-302919).

\section{REFERENCES}

[1] Johnson I. P., Webb D. J., Kalli K., Large M. C. and Argyros A., "Multiplexed FBG sensor recorded in multimode microstructured polymer optical fibre". In: Kalli K. and Urbanczyk W., editors. Photonic Crystal Fibres; 14-16 April; Brussels - Photonics Europe: SPIE vol 7714, p. 10. (2010)

[2] Abang, A. and Webb, D. J., "Demountable connection for polymer optical fiber grating sensors," Optical Engineering, 51(8) 080503 (2012).

[3] Abdi O., Wong K. C., Hassan T., Peters K. J. and Kowalsky M. J., “Cleaving of single mode polymer optical fiber for strain sensor applications", Opt. Commun. 282 856-61 (2009).

[4] W. Zhang et al., "Investigation into time response of polymer fibre Bragg grating based humidity sensors," Journal of Lightwave Technology, 30(8), 1090-1096 (2012).

[5] Thorlabs Inc., "Guide to connectorization and polishing optical fibres," Fibre polishing notes, June 2012 , http://www.thorlabs.com/Thorcat/1100/1166-D02.pdf (11 May 2012).

[6] N. A. Mortensen, J. R. Folkenberg ,M. D. Nielsen, K. P. Hansen " Modal cutoff and the $V$ parameter in photonic crystal fibers" Vol. 28, No. 20, Optics Letters, (2003).

[7] A. Stefani, K. Nielsen, H.K. Rasmussen, O. Bang, "Cleaving of TOPAS and PMMA microstructured polymer optical fibers: Core-shift and statistical quality optimization”, Opt. Commun. 285, 1825-1833 (2012). 